\title{
Erratum
}

\section{Estimates for the asymptotic behavior of solutions of the Helmholtz equation, with an application to second order elliptic differential operators with variable coefficients}

\section{H.D. Alber}

Technische Hochschule Darmstadt, Fachbereich Mathematik, Schlossgartenstrasse 7 , W-6100 Darmstadt, Federal Republic of Germany

\section{Math. Z. 167, 213-226 (1979)}

It has been pointed out to the author by $N$. Weck that the proof of the estimates (A6) and (A7) in the case $r>p>0$ is not correct, since the estimates (A4) and (A5) yield a remainder term $r^{1 / 2} O\left(p^{-4 / 3}\right)$, which is not uniformly bounded in $r$. Instead, the estimates (A6) and (A7) must be proved as follows:

The proof given in the paper is correct for all $r$ and $p$ with $p<r \leqq C\left(p+p^{3}\right)$. For $r \geqq \frac{1}{2}\left(p+\frac{3}{2}\right)\left(p-\frac{1}{2}\right)>0$ we use the integral representation

$$
H_{p}^{(1)}(r)=\frac{1}{\Gamma(p+1 / 2)} \sqrt{\frac{2}{\pi r}} e^{i\left(r-(p+1 / 2) \frac{\pi}{2}\right)} \int_{0}^{\infty} e^{-t} t^{p-1 / 2}\left(1+\frac{i t}{2 r}\right)^{p-1 / 2} d t
$$

(cf. [1, p. 6 and p. 23]), and obtain

$$
\begin{aligned}
\left|r^{1 / 2} H_{p}^{(1)}(r)\right| & \leqq\left(\frac{2}{\pi}\right)^{1 / 2} \frac{1}{\Gamma(p+1 / 2)} \int_{0}^{\infty} e^{-t} t^{p-1 / 2}\left(1+\frac{t}{2 r}\right)^{p-1 / 2} d t \\
& \leqq\left(\frac{2}{\pi}\right)^{1 / 2} \frac{1}{\Gamma(p+1 / 2)} \int_{0}^{\infty} e^{-t} t^{p-1 / 2} e^{(p-1 / 2) \frac{t}{2 r}} d t \\
& \leqq\left(\frac{2}{\pi}\right)^{1 / 2} \frac{1}{\Gamma(p+1 / 2)}\left[\frac{1}{1-\frac{p-1 / 2}{2 r}}\right]^{p+1 / 2} \int_{0}^{\infty} e^{-\tau} \tau^{p-1 / 2} d \tau \\
& =\left(\frac{2}{\pi}\right)^{1 / 2}\left[\frac{1}{1-\frac{p-1 / 2}{2 r}}\right]^{p+1 / 2} \leqq\left(\frac{2}{\pi}\right)^{1 / 2}\left(1+\frac{1}{p+1 / 2}\right)^{p+1 / 2} \leqq\left(\frac{2}{\pi}\right)^{1 / 2} e
\end{aligned}
$$

since

$$
\frac{1}{1-\frac{p-1 / 2}{2 r}} \leqq 1+\frac{1}{p+1 / 2} \quad \text { for } r \geqq \frac{1}{2}\left(p+\frac{3}{2}\right)\left(p-\frac{1}{2}\right)
$$


For these $r$ and $p$ we also have

$$
\left|r^{1 / 2} J_{p}(r)\right| \leqq\left(\frac{2}{\pi}\right)^{1 / 2} e
$$

which is obtained from a similar estimate for $H_{p}^{(2)}$ and from $J_{p}(r)=\frac{1}{2}\left(H_{p}^{(1)}(r)\right.$ $\left.+H_{p}^{(2)}(\boldsymbol{r})\right)$.

\section{Reference}

1. Erdélyi, A., Magnus, W., Oberhettinger, F., Tricomi, F.: Higher transcendental functions, vol. 2. New York: McGraw Hill 1953 